\title{
Investigation of potential non-HLA rheumatoid arthritis susceptibility loci in a European cohort increases the evidence for nine markers
}

\author{
Darren Plant, ${ }^{1}$ Edward Flynn, ${ }^{1}$ Hamdi Mbarek, ${ }^{2}$ Philippe Dieudé, ${ }^{2}$ François Cornelis, ${ }^{2}$ \\ Lisbeth Ärlestig, ${ }^{3}$ Solbritt Rantapää Dahlqvist, ${ }^{3}$ George Goulielmos, ${ }^{4}$ \\ Dimitrios T Boumpas, ${ }^{4}$ Prodromos Sidiropoulos, ${ }^{4}$ Julia S Johansen, ${ }^{5}$ \\ Lykke M Ørnbjerg, ${ }^{5}$ Merete Lund Hetland, ${ }^{5}$ Lars Klareskog, ${ }^{6}$ Andrew Filer, ${ }^{7,8}$ \\ Christopher D Buckley, ${ }^{7,8}$ Karim Raza, ${ }^{7,8}$ Torsten Witte, ${ }^{9}$ Reinhold E Schmidt, ${ }^{9}$ \\ Jane Worthington ${ }^{1}$
}

- Additional data are published online only. To view these files please visit the journal online (http://ard.bmj.com)

1 arc-Epidemiology Unit, University of Manchester, Manchester, UK

2Department of Rheumatology, Evry University, Paris, France

${ }^{3}$ Department of Rheumatology, University Hospital, Umeå, Sweden

${ }^{4}$ Clinic of Rheumatology, Department of Medicine University of Crete, Heraklion, Greece

${ }^{5}$ DANBIO, Copenhagen University Hospital at Hvidovre, Copenhagen, Denmark ${ }^{6}$ Department of Medicine, Karolinska Institutet, Karolinska University Hospital, Stockholm, Sweden

${ }^{7}$ Department of Rheumatology, Sandwell and West Birmingham Hospitals NHS Trust, Birmingham, UK

${ }^{8}$ Rheumatology Research Group, The University of Birmingham, Birmingham, UK

${ }^{9}$ Department of Immunology and Rheumatology, Hannove Medical School, Hannover, Germany

\section{Correspondence to}

Dr Darren Plant, arcEpidemiology Unit, Stopford Building, The University of Manchester, Manchester M13 9PT, UK:

darren.plant@manchester.ac.uk

Accepted 31 January 2010

\section{ABSTRACT}

Background Genetic factors have a substantial role in determining development of rheumatoid arthritis (RA), and are likely to account for $50-60 \%$ of disease susceptibility. Genome-wide association studies have identified non-human leucocyte antigen RA susceptibility loci which associate with RA with low-to-moderate risk. Objectives To investigate recently identified RA susceptibility markers using cohorts from six European countries, and perform a meta-analysis including previously published results.

Methods 3311 DNA samples were collected from patients from six countries (UK, Germany, France, Greece, Sweden and Denmark). Genotype data or DNA samples for 3709 controls were collected from four countries (not Sweden or Denmark). Eighteen single nucleotide polymorphisms (SNPS) were genotyped using Sequenom MassArray technology. Samples with a $>95 \%$ success rate and only those SNPs with a genotype success rate of $>95 \%$ were included in the analysis. Scandinavian patient data were pooled and previously published Swedish control data were accessed as a comparison group. Meta-analysis was used to combine results from this study with all previously published data.

Results After quality control, 3209 patients and 3692 controls were included in the study. Eight markers (ie, rs1160542 (AFF3), rs1678542 (KIF5A), rs2476601 (PTPN22), rs3087243 (CTLA4), rs4810485 (CD40), rs5029937 (6q23), rs10760130 (TRAF1/C5) and rs7574865 (STAT4)) were significantly associated with RA by meta-analysis. All 18 markers were associated with RA when previously published studies were incorporated in the analysis. Data from this study increased the significance for association with RA and nine markers. Conclusions In a large European RA cohort further evidence for the association of 18 markers with RA development has been obtained.

\section{INTRODUCTION}

Genetic factors have a substantial role in determining development of rheumatoid arthritis (RA), and are likely to account for $50-60 \%$ of disease susceptibility. ${ }^{1}$ In patients of European ancestry, the strongest genetic effects are conferred by the HLA-DRB1 locus on chromosome $6,^{2}$ (specifically the alleles encoding the shared epitope) and the protein tyrosine phosphatase 22 (PTPN22) gene on chromosome $1 \mathrm{p} 13 .^{3}$

Recently, a number of potential RA genetic susceptibility loci, associating with RA susceptibility with low-to-moderate risk, have been identified through genome-wide association studies. These loci including 6q23, TRAF1/C5, STAT4, IL2RB, KIF5A, PRKCQ IL2/IL21, CD226, CCL21, CD40, CTLA4 and IL2RA, defined by single nucleotide polymorphisms (SNPs), have subsequently been replicated in large independent panels of patients and healthy controls. ${ }^{4-20}$ Although these investigations substantially improve our understanding of the genetic component of RA susceptibility in patients of European ancestry, they have been limited to UK, US, Dutch and Swedish patients.

AutoCure is an EU-funded research project within the sixth framework programme (http:// www.autocure.org, accessed 25 March 2010). The project includes 26 different partners from 12 countries-from Umeå in the north of Sweden to Crete in southern Greece. In AutoCure we have assembled a large panel of samples from patients with RA and controls from six countries (UK, Germany, France, Greece, Sweden and Denmark): the Genetic Repository for AutoCure (GRACE) collection, in which we can now investigate disease susceptibility loci. The aim of this study was therefore to investigate the recently identified RA susceptibility SNPs using RA cohorts from these six European countries, and perform a metaanalysis of these data together with previously published results.

\section{METHODS}

\section{Cohorts}

Researchers working within partner institutes identified DNA samples from patient and control subjects and a unique anonymised identifier was assigned to each sample. Patients were Caucasian of European descent and fulfilled the 1987 American College of Rheumatology classification criteria for genetic studies. ${ }^{21} 22$ DNA samples were received at the Arthritis Research Campaign Epidemiology Unit (arc-EU), within the University of Manchester, Manchester, UK. DNA samples were stored at $-20^{\circ} \mathrm{C}$ before genotyping 
and were managed on a fully integrated laboratory information management system. A total of 3311 patient DNA samples were collected from six countries (Manchester and Birmingham, UK, n=1199; Hannover, Germany, n=222; Paris, France, $n=727$; Crete, Greece, $\mathrm{n}=300$; Umeå, Sweden, $\mathrm{n}=567$; Copenhagen, Denmark, $\mathrm{n}=296$ ) and healthy control DNA samples were collected from Germany, $n=217$; France, $n=192$; and Greece, $\mathrm{n}=300$. Control genotype data for the UK cohort $(\mathrm{n}=3000)$ were extracted from the Wellcome Trust Case-Control Consortium 2 (WTCCC2) EGA website. Patient demographic and serological information was gathered where collected. Ethical approval was obtained from each individual centre to contribute samples to the GRACE collection and to be used in genetic association studies.

\section{Genotyping and analysis}

Eighteen SNPs were selected for genotyping using Sequenom Mass Array technology according to the manufacturer's instructions, using iPLEX chemistry (http://www.sequenom.com, accessed 25 March 2010). The SNPs were markers associated with RA susceptibility in cohorts tested by our group and others and consisted of: rs1160542 (AFF3), rs2812378 (CCL21), rs763361 (CD226), rs4810485 (CD40), rs3087243 and rs231775 (CTLA4), rs6822844 (IL2/IL21), rs2104286 (IL2RA), rs743777 (IL2RB), rs1678542 (KIF5A), rs4750316 (PRKCQ), rs7574865 (STAT4), rs13207033, rs5029937 and rs6920220 (6q23), rs10760130 and rs2900180 (TRAF1/C5) and rs2476601 (PTPN22).

Only those samples with a $>95 \%$ success rate and only those SNPs with a genotype success rate of $>95 \%$ were included in the analysis. Allele frequencies were calculated in both patient and control groups for each SNP marker, and meta-analysis was used to combine results for all cohorts.

Control samples were not received from Umeå or Copenhagen. In order to maximise the number of samples available for meta-analysis, we investigated the possibility of combining data from the two Scandinavian centres and comparing this with previously published Swedish control data from the Epidemiological Investigation of Rheumatoid Arthritis (EIRA) cohort. ${ }^{13}$ The $\chi^{2}$ test statistic was used to assess (at the $5 \%$ significance threshold) differences in allele frequency for each SNP between Swedish and Danish patients' data and only where SNP frequencies were not significantly different was data combined. EIRA control data were available for 16 of the 18 SNPs under investigation (not rs231775 or rs5029937).

Finally, a meta-analysis incorporating GRACE samples with previously published data for each SNP was performed. Previously published data for the SNPs under investigation were retrieved from the literature and scrutinised to ensure that no overlapping samples were included in the analyses. Fixed effects models (Mantel-Haenszel method) were used and studies were weighted according to the amount of information they contained. Cochran's $Q$ and $I^{2}$ test statistics were used to estimate variation in effects attributable to heterogeneity, and $\chi^{2} p$ values are presented. Where the heterogeneity was significant (at the 5\% significance threshold), random effects models (DerSimonian and Laird method) were used, where stated, to incorporate betweenstudy variation in the weighting.

\section{Power calculation}

Sample size and power were calculated based on previously published effect sizes and are presented at the $5 \%$ significance level.

All analyses were performed using STATA statistical software (StataCorp 2005: release 9; College Station, Texas, USA).

\section{RESULTS}

\section{Cohort characteristics and quality control}

Demographic, clinical and laboratory information for each patient cohort are presented in table 1 . After removal of samples that did not satisfy quality control, 3209 patient and 3692 control samples remained available for analysis (success rate, 98\%). SNP markers satisfied Hardy-Weinberg equilibrium in healthy controls (at the $5 \%$ significance threshold), with the exception of the following minor deviation: rs6920220 (6q23) UK controls; $\mathrm{p}=0.02$ (online supplementary table 1 ).

\section{Comparison of allele frequencies in centre-matched case control cohorts}

For the UK, German, French and Greek centres, where both patients and controls were collected, allelic odds ratios (ORs) were calculated to test for the association of SNP markers with RA (online supplementary table 1). None of the SNPs associated with RA across all cohorts. However, the SNP markers rs2812378 (CCL21) Hannover, rs3087243 (CTLA4) and rs743777 (IL2RB) Paris, rs2900180, rs10760130 (TRAF1/ C5) and rs13207033 (6q23) Manchester, rs2476601 (PTPN22) Manchester, Paris and Hannover, rs5029937 (6q23) Manchester and Crete, and rs4810485 (CD40) Crete and Hannover, significantly associated with RA (online supplementary table 1). A meta-analysis of the data from these four centres showed that five SNP markers rs6920220, rs2900180, rs10760130, rs2476601 and rs5029937 associated with RA (online supplementary table 1).

\section{Meta-analysis of the GRACE cohort, including EIRA control data}

In order to include all patient data generated in this study in a meta-analysis we accessed previously published control data from the EIRA study, ${ }^{13}$ as a comparison group for Scandinavian patients. EIRA control data comprised: imputed data for eight loci from 660 healthy subjects and genotype data from up to 900 healthy subjects for eight loci (online supplementary table 2). No Swedish control data were available for the SNP markers rs231775 or rs5029937.

Table 1 Demographic and laboratory characteristics of GRACE cohorts

\begin{tabular}{llllllll}
\hline $\begin{array}{l}\text { Cohort } \\
\text { characteristics }\end{array}$ & Manchester & Birmingham & Umeå & Copenhagen & Paris & Crete & Hannover \\
\hline N & 1087 & 112 & 567 & 296 & 727 & 300 & 222 \\
AAO (years \pm SD) & $54.5 \pm 15.0$ & $57.0 \pm 14.9$ & $55.9 \pm 14.4$ & $48.1 \pm 15.4$ & - & $55.4 \pm 4.3$ & $45.3 \pm 11.9$ \\
Female (\%) & 68 & 58 & 67 & 75 & - & 74 & 77 \\
Anti-CCP+ (\%) & 37 & 60 & 68 & 61 & - & & 71 \\
RF+ (\%) & 38 & 69 & 72 & 66 & - & 43 & 78 \\
\hline
\end{tabular}

$\mathrm{AAO}$, mean age at onset of symptom with $\mathrm{SD}$; anti-CCP+, percentage of patients with a positive anti-cyclic citrullinated peptide titre; GRACE, Genetic Repository for AutoCure; N, number of observations; RF+, number of patients with a positive rheumatoid factor titre. 
Allele frequencies for Swedish and Danish patients were combined for 15 of the 16 SNPs listed in online supplementary table 2. The SNP marker rs2476601 differed sufficiently in allele frequency between Swedish and Danish patients (at the 5\% significance threshold) and was therefore not combined (data not shown; Swedish data alone contributed to the meta-analysis for this SNP).

In the meta-analysis incorporating GRACE and EIRA control data, eight of the 18 SNPs investigated were significantly associated with RA (at the $5 \%$ significance threshold) by meta-analysis (ie, rs1160542 (AFF3), rs1678542 (KIF5A), rs2476601 (PTPN22), rs3087243 (CTLA4), rs4810485 (CD40), rs5029937 (6q23), rs10760130 (TRAF1/C5) and rs7574865 (STAT4); table 2). One marker associated with RA in the samples from Paris (rs743777) and Hannover (rs2812378)$\mathrm{OR}=1.31(95 \%$ CI 1.00 to 1.71$)$ and $\mathrm{OR}=1.42(95 \%$ CI 1.07 to 1.88 ), respectively (online supplementary table 1 ), but did not associate with RA by meta-analysis-OR=1.07 (95\% CI 0.91 to 1.26 ) and 1.04 (95\% CI 0.97 to 1.13 ), respectively. This observation is likely to be explained by lack of power. We had $<80 \%$ power to detect an effect size in keeping with that observed in the original studies. ${ }^{14} 17$

Between-study heterogeneity was observed for the SNP markers rs2476601 (PTPN22; $\mathrm{p}=0.01)$, rs4810485 (CD40; $\mathrm{p}=0.01$ ) and rs7574865 (STAT4; $\mathrm{p}=0.03$ ). These observations were due to the very strong effect seen for rs2476601 in the cohorts from Paris and Hannover $(\mathrm{OR}=2.47$ (95\% CI 1.63 to 3.73$))$ and $(\mathrm{OR}=2.05$ (95\% CI 1.40 to 3.02$)$ ), respectively, and a lack of association observed in the cohort from Crete $(\mathrm{OR}=1.06$ (95\% CI 0.56 to 2.00)); a lack of effect seen in the UK (OR=1.02 (95\% CI 0.91 to 1.14$)$ ) and Paris $(\mathrm{OR}=1.16(95 \%$ CI 0.86 to 1.56$))$ cohorts for rs4810485; and the strong effect seen for rs7574865 in the Swedish cohort (OR=1.44 (95\% CI 1.23 to 1.68); online supplementary table 1). The marker rs7574865 did not significantly associate with RA in any other cohort tested (online supplementary table 1).

\section{Meta-analysis of all available data, including previously published data}

A further meta-analysis including data from this study and all previously published data for the 18 SNPs under investigation was performed. Where available, allele frequency data were retrieved from the literature, or were requested by personal communication from the relevant primary investigator (online supplementary table 3). All 18 SNPs significantly associated with RA in the meta-analysis (table 3). Significant between-study heterogeneity was observed for the SNP markers rs743777 (IL2RB), rs2476601 (PTPN22), rs2900180 and rs10760130 (TRAF1/C5). Sensitivity testing showed that the between-study heterogeneity resulted from the strong effect at rs743777 in the original WTCCC discovery cohort. ${ }^{16}$ No between-study heterogeneity was observed after removal of this study from the meta-analysis $(p=0.08)$. The between-study heterogeneity identified for rs2476601 ( $p=0.0003$ ), rs2900180 $(\mathrm{p}=1.69 \mathrm{e}-06)$ and rs10760130 ( $\mathrm{p}=9.62 \mathrm{e}-06)$ resulted from broad differences in effect sizes between individual study cohorts. For rs2476601 the effect was seen in the same direction for all cohorts considered, apart from the cohort originating from Crete, Greece (figure 1).

\section{DISCUSSION}

Through collaboration between European partner institutes within the AutoCure consortium (http://www.autocure.org), we were able to collect a large replication cohort of DNA samples from patients with RA and healthy controls, from six countries (UK, Germany, France, Greece, Sweden and Denmark). In a meta-analysis of four centres where both patients and controls were available we were able to replicate the association of five SNP markers with RA susceptibility, in a pan-European setting (ie, rs6920220, rs2900180, rs10760130, rs2476601 and rs5029937). Patients from Scandinavian cohorts were not included in this meta-analysis.

When allele counts for Scandinavian patients were compared with previously published EIRA control data the SNP markers rs 1678542 (OR=0.81 (95\% CI 0.70 to 0.94$))$, rs4810485 ( $\mathrm{OR}=0.78$ (95\% CI 0.66 to 0.93$)$ ), rs $2476601(\mathrm{OR}=1.54$ (95\% CI 1.24 to 1.90$)$ ) and $\mathrm{rs} 7574865(\mathrm{OR}=1.44(95 \% \mathrm{CI} 1.23$ to 1.68$)$ ) associated with RA (results not shown). In a meta-analysis incorporating all GRACE material together with previously published EIRA controls we observed associations with eight SNP markers and RA susceptibility (table 2). When SNP associations with RA were replicated in this meta-analysis, the

Table 2 Meta-analysis results for AutoCure samples-GRACE collection including EIRA control data

\begin{tabular}{|c|c|c|c|c|c|c|c|}
\hline SNP & Gene & Case/control & MAF case & MAF control & Het p & $\chi^{2} \mathbf{p}$ & OR (95\% Cl) \\
\hline rs231775 & CTLA4 & $2272 / 3317$ & 0.37 & 0.38 & 0.071 & 0.647 & $1.02(0.94$ to 1.11$)$ \\
\hline rs743777 & IL2RB & $3068 / 4048$ & 0.33 & 0.32 & 0.086 & 0.408 & $1.07(0.91$ to 1.26$)$ \\
\hline rs763361 & CD226 & $3152 / 3992$ & 0.49 & 0.47 & 0.782 & 0.396 & $1.03(0.96$ to 1.11$)$ \\
\hline rs1160542 & AFF3 & $3146 / 4062$ & 0.49 & 0.47 & 0.854 & 0.029 & $1.08(1.01$ to 1.16$)$ \\
\hline rs1678542 & KIF5A & $3144 / 4065$ & 0.36 & 0.38 & 0.073 & 0.023 & $0.92(0.86$ to 0.99$)$ \\
\hline rs2104286 & IL2RA & $3149 / 4066$ & 0.24 & 0.26 & 0.898 & 0.115 & $0.94(0.86$ to 1.01$)$ \\
\hline rs2476601 & PTPN22 & $2794 / 4194$ & 0.15 & 0.10 & 0.010 & $6.25 e-12$ & $1.48(1.33$ to 1.66$)$ \\
\hline rs2812378 & CCL21 & $3146 / 4062$ & 0.35 & 0.33 & 0.187 & 0.251 & $1.04(0.97$ to 1.13$)$ \\
\hline rs2900180 & TRAF1/C5 & $3147 / 4223$ & 0.36 & 0.35 & 0.095 & 0.057 & $1.07(0.99$ to 1.15$)$ \\
\hline rs3087243 & CTLA4 & $3058 / 4279$ & 0.42 & 0.44 & 0.279 & 0.024 & $0.92(0.86$ to 0.99$)$ \\
\hline rs4750316 & PRKCQ & $3075 / 4046$ & 0.18 & 0.19 & 0.525 & 0.501 & $0.97(0.86$ to 1.06$)$ \\
\hline rs4810485 & CD40 & $3043 / 4044$ & 0.24 & 0.25 & 0.010 & 0.033 & $0.91(0.84$ to 0.99$)$ \\
\hline rs5029937 & $6 q 23$ & $2262 / 3379$ & 0.04 & 0.03 & 0.166 & 0.002 & $1.40(1.13$ to 1.74$)$ \\
\hline rs6822844 & IL2/IL21 & $3143 / 3998$ & 0.16 & 0.16 & 0.329 & 0.656 & $0.98(0.89$ to 1.07$)$ \\
\hline rs6920220 & $6 q 23$ & $3146 / 4066$ & 0.21 & 0.21 & 0.479 & 0.093 & $1.08(0.99$ to 1.17$)$ \\
\hline rs7574865 & STAT4 & $3063 / 4198$ & 0.25 & 0.22 & 0.030 & 0.0003 & $1.17(1.07$ to 1.27$)$ \\
\hline rs10760130 & TRAF1/C5 & $3155 / 4254$ & 0.45 & 0.43 & 0.348 & 0.035 & $1.08(1.01$ to 1.16$)$ \\
\hline rs13207033 & $6 q 23$ & $2984 / 4042$ & 0.26 & 0.26 & 0.153 & 0.348 & $0.96(0.89$ to 1.04$)$ \\
\hline
\end{tabular}




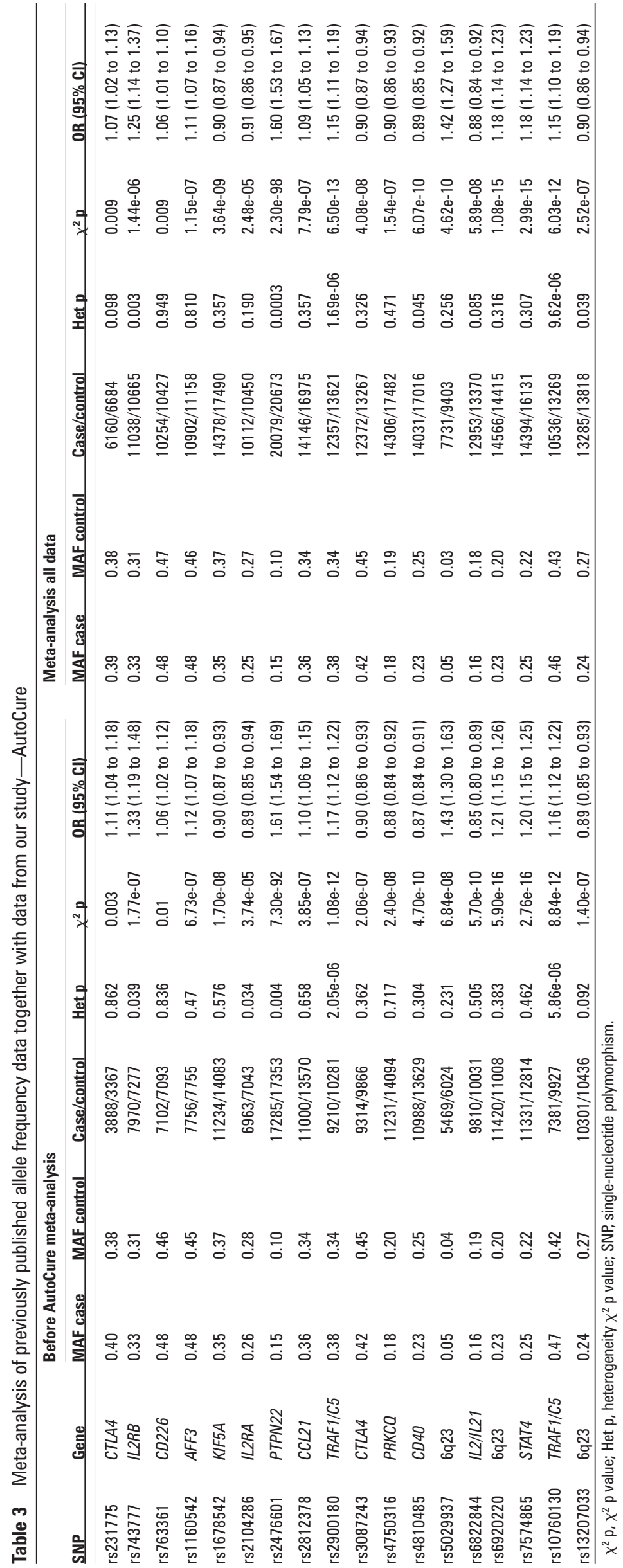


rs2476601

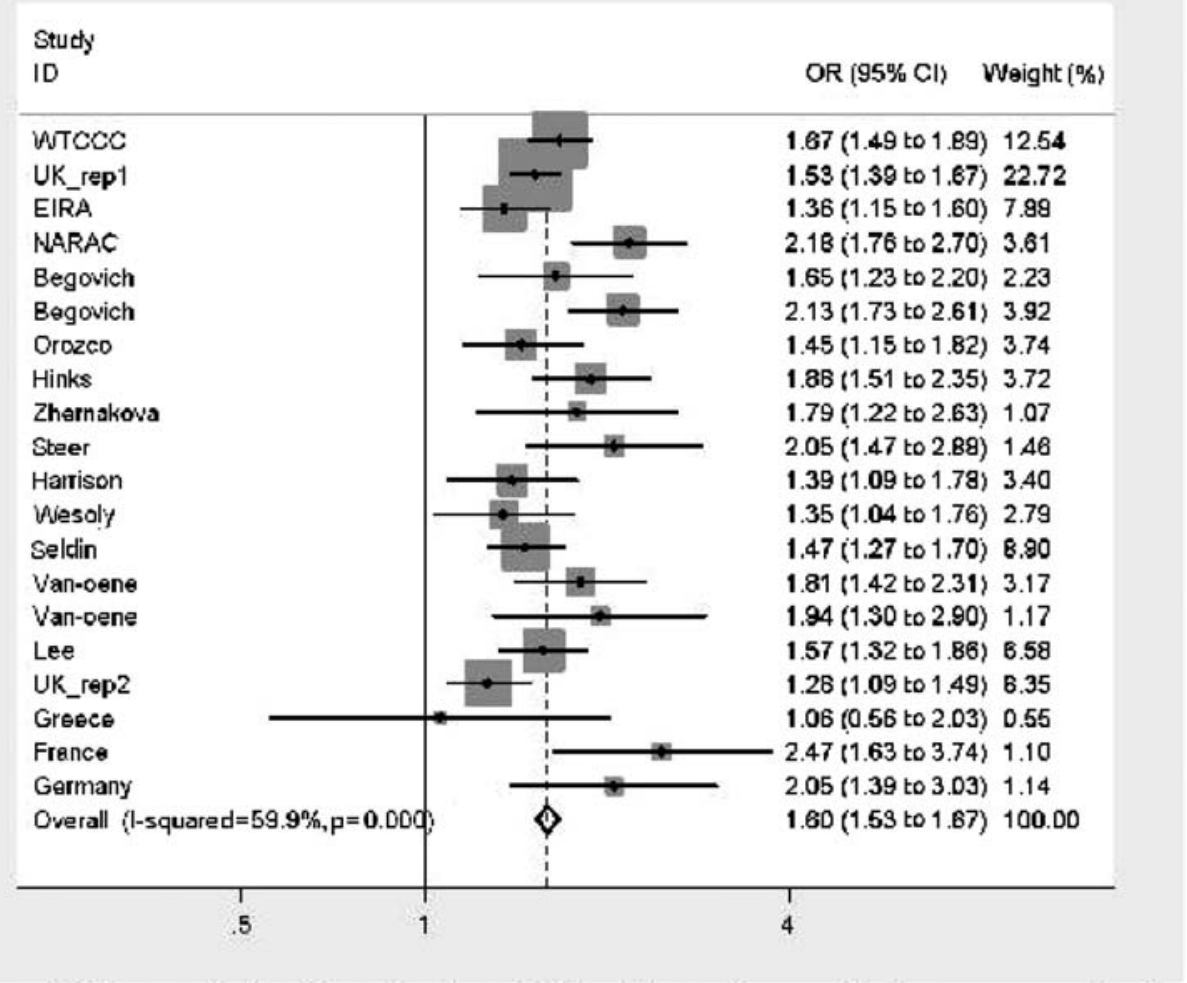

Figure 1 Meta-analysis of previously published data, together with data generated in this study (labelled: UK_rep2 (UK), Greece, France, Germany and EIRA for Scandinavian cohorts) for the single-nucleotide polymorphism maker rs2476601 (PTPN22). Odds ratio (OR) point estimate and 95\% Cl (horizontal line) for each cohort are shown. The diamond represents the meta-analysis point estimate and $\mathrm{Cl}$. The scale of the effect size is given on the $x$-axis. EIRA, Epidemiological Investigation of Rheumatoid Arthritis; WTCCC, Wellcome Trust Case Control Consortium.

results were comparable to those previously reported. ${ }^{3-5} 14$ Where SNPs failed to replicate we had between $10 \%$ and $93 \%$ power to detect an effect size of the same magnitude previously reported.

The well-established RA susceptibility SNP within PTPN22 (rs2476601) appeared not to associate with RA in patients from Crete, Greece. This might be owing to a lack of power resulting from small sample size. Given the minor allele frequency of the PTPN22 variant in Crete control samples (3\%), 5000 patient and control samples would be required to detect even a large effect at this locus-that is, a $50 \%$ increase in risk with $80 \%$ power at $p=0.05$. The minor allele for rs 2476601 was observed at low frequency in patients from Crete (4\%), similar to that seen in the Turkish population (3\%)-where no association with RA has been detected. ${ }^{23}$ There remains no supportive evidence for a significant effect at the PTPN22 locus in SouthEastern European patients with RA.

In the meta-analysis incorporating previously published results, all 18 SNPs significantly associated with RA susceptibility. The association of nine SNPs (rs231775, rs763361, rs1160542, rs1678542, rs2104286, rs2476601, rs2900180, rs3087243 and rs5029937) increased in significance by inclusion of GRACE cohort data (table 3). Significant between-study heterogeneity was observed for SNPs at PTPN22 (figure 1). Applying a random effects model to the meta-data showed that the mean OR for rs2476601 was 1.64 (95\% CI 1.52 to 1.77). Between-study variation in effect size was also seen for the TRAF1/C5 SNPs. Applying random effects models at the
TRAF1/C5 locus showed that the mean OR was 1.15 (95\% CI 1.05 to 1.26$)$ and 1.16 (95\% CI 1.07 to 1.26$)$ for rs 2900180 and rs10760130, respectively. Between-study heterogeneity at the TRAF1/C5 locus has previously been reported. ${ }^{24}$ Underlying differences in effects may be reflective of population stratification. We tried to avoid a pooling strategy in the meta-analysis where possible; however, the Swedish and Danish patient samples were pooled owing to a lack of healthy controls for these patients.

A weakness in this study was that ancestry informative markers were not available to more formally assess genetic differences due to differences in geographical location.

There is emerging evidence that different genetic effects exist in serologically defined patient groups. ${ }^{2526}$ We performed metaanalyses of genetic markers in serologically defined patients within GRACE, in which we analysed anti-citrullinated protein antibody (ACPA)-positive patients separately (online supplementary table 4). The number of observations in the subanalyses was modest; however, eight SNP markers were associated with ACPA-positive RA: rs1160542 (AFF3), rs1678542 (KIF5A), rs2476601 (PTPN22), rs2900180 and rs10760130 (TRAF1/C5) rs4810485 (CD40), rs5029937 (6q23) and rs7574865 (STAT4). For SNPs that were associated with RA in the ACPA-positive subgroup, the effect was predominantly greater (table 2 and online supplementary table 4). The current meta-analysis of GRACE data and previously published studies included data from cohorts of anti-cyclic citrullinated peptide-positive patients with RA, ${ }^{13}$ and cohorts in which no formal subdivision 
had been performed. ${ }^{16}$ This meta-analysis is therefore heterogeneous with respect to seropositivity and future studies taking account of subphenotypes may disclose different genetic associations.

In this large pan-European collaboration we have established a replication cohort of up to 7000 patients and controls from six countries. In an initial experiment using this material we provide additional evidence to support 18 loci as susceptibility markers for RA in people of European ancestry.

Acknowledgements For helpful genotyping advice the authors would like to thank Dr Steve Eyre, arc-Epidemiology Unit, University of Manchester, UK. This study makes use of data generated by the Wellcome Trust Case-Control Consortium 2. A full list of the investigators who contributed to the generation of the data is available from http://www.wtccc.org.uk.

Funding Provided by the European Community's Sixth Framework Programme AutoCure and by the Wellcome Trust under award 085475.

Competing interests None.

Ethics approval This study was conducted with the approval of each individual centre.

Provenance and peer review Not commissioned; externally peer reviewed.

\section{REFERENCES}

1. MacGregor AJ, Snieder H, Rigby AS, et al. Characterizing the quantitative genetic contribution to rheumatoid arthritis using data from twins. Arthritis Rheum 2000;43:30-7.

2. Gregersen PK, Silver J, Winchester RJ. The shared epitope hypothesis. An approach to understanding the molecular genetics of susceptibility to rheumatoid arthritis. Arthritis Rheum 1987;30:1205-13.

3. Begovich AB, Carlton VE, Honigberg LA, et al. A missense single-nucleotide polymorphism in a gene encoding a protein tyrosine phosphatase (PTPN22) is associated with rheumatoid arthritis. Am J Hum Genet 2004;75:330-7.

4. Barton A, Thomson W, Ke X, et al. Re-evaluation of putative rheumatoid arthritis susceptibility genes in the post-genome wide association study era and hypothesis of a key pathway underlying susceptibility. Hum Mol Genet 2008;17:2274-9.

5. Barton A, Thomson W, Ke X, et al. Rheumatoid arthritis susceptibility loci at chromosomes 10p15, 12q13 and 22q13. Nat Genet 2008;40:1156-9.

6. Bowes $\mathbf{J}$, Barton A. Recent advances in the genetics of RA susceptibility. Rheumatology (Oxford) 2008;47:399-402.

7. Daha NA, Kurreeman FA, Marques RB, et al. Confirmation of STAT4, IL2/ IL21, and CTLA4 polymorphisms in rheumatoid arthritis. Arthritis Rheum 2009;60:1255-60.
8. Hafler JP, Maier LM, Cooper JD, et al. CD226 Gly307Ser association with multiple autoimmune diseases. Genes Immun 2009;10:5-10.

9. Kurreeman FA, Padyukov L, Marques RB, et al. A candidate gene approach identifies the TRAF1/C5 region as a risk factor for rheumatoid arthritis. PLoS Med 2007; 4:e278.

10. Orozco G, Alizadeh BZ, Delgado-Vega AM, et al. Association of STAT4 with rheumatoid arthritis: a replication study in three European populations. Arthritis Rheum 2008;58:1974-80.

11. Plenge RM, Padyukov L, Remmers EF, et al. Replication of putative candidate-gene associations with rheumatoid arthritis in $>4,000$ samples from North America and Sweden: association of susceptibility with PTPN22, CTLA4, and PADI4. Am J Hum Genet 2005;77:1044-60.

12. Plenge RM, Cotsapas C, Davies L, et al. Two independent alleles at $6 q 23$ associated with risk of rheumatoid arthritis. Nat Genet 2007;39:1477-82.

13. Plenge RM, Seielstad M, Padyukov L, et al. TRAF1-C5 as a risk locus for rheumatoid arthritis - a genomewide study. N Engl J Med 2007;357:1199-209.

14. Raychaudhuri S, Remmers EF, Lee AT, et al. Common variants at CD40 and other loci confer risk of rheumatoid arthritis. Nat Genet 2008; 40:1216-23.

15. Remmers EF, Plenge RM, Lee AT, et al. STAT4 and the risk of rheumatoid arthritis and systemic lupus erythematosus. N Engl J Med 2007;357:977-86.

16. The Wellcome Trust Case Control Consortium. Genome-wide association study of 14,000 cases of seven common diseases and 3,000 shared controls. Nature 2007:447:661-78.

17. Thomson W, Barton A, Ke X, et al. Rheumatoid arthritis association at 6q23. Nat Genet 2007;39:1431-3.

18. Zhernakova A, Eerligh P, Barrera P, et al. CTLA4 is differentially associated with autoimmune diseases in the Dutch population. Hum Genet 2005;118:58-66.

19. Zhernakova A, Eerligh P, Wijmenga $\mathrm{C}$, et al. Differential association of the PTPN22 coding variant with autoimmune diseases in a Dutch population. Genes Immun 2005;6:459-61.

20. Zhernakova A, Alizadeh BZ, Bevova M, et al. Novel association in chromosome 4q27 region with rheumatoid arthritis and confirmation of type 1 diabetes point to a general risk locus for autoimmune diseases. Am J Hum Genet 2007;81:1284-8.

21. Arnett FC, Edworthy SM, Bloch DA, et al. The American Rheumatism Association 1987 revised criteria for the classification of rheumatoid arthritis. Arthritis Rheum 1988;31:315-24.

22. MacGregor AJ, Bamber S, Silman AJ. A comparison of the performance of different methods of disease classification for rheumatoid arthritis. Results of an analysis from a nationwide twin study. J Rheumatol 1994;21:1420-6.

23. Sahin N, Gunduz F, Inanc N, et al. No association of PTPN22 gene polymorphism with rheumatoid arthritis in Turkey. Rheumatol Int 2009 Apr 9. [Epub ahead of print].

24. Patsopoulos NA, loannidis JP. Susceptibility variants for rheumatoid arthritis in the TRAF1-C5 and 6q23 loci: a meta-analysis. Ann Rheum Dis 2010;69:561-6.

25. Klareskog L, Catrina Al, Paget S. Rheumatoid arthritis. Lancet 2009;373:659-72.

26. van der Helm-van Mil AH, Huizinga TW, de Vries RR, et al. Emerging patterns of risk factor make-up enable subclassification of rheumatoid arthritis. Arthritis Rheum 2007; 56:1728-35. 